\title{
Advances in Broad Ion Beam Processing for 3D Microscopy and Microanalysis of Ceramic Composites
}

\author{
W. Hauffe* and R.J. Mitro** \\ *Physics Department, Dresden University of Technology, D-01062 Dresden, Germany \\ **Gatan R\&D, 5933 Coronado Lane, Pleasanton, CA 94588, USA
}

Ion beam processing of heterogeneous samples allows investigating three-dimensional microstructures by microscopy and microanalysis [1]. The possible methods and standard processing steps have been reported earlier including the applicability of available ion beam instruments and typical applications (e.g. in metallography and microelectronic) were demonstrated [2]. All materials including composites with strong differences in hardness can be investigated by sputtering. Ceramic materials require special processing to reveal the internal microstructure without producing artefacts. In most cases a combination of different ion beam processing steps according to FIG. 1 is necessary. Ion beam slope cutting (IBSC) up to the required depth is the main process [1]. The developed slope as a representative cut without selective etching effects can be observed by atomic number contrast in the SEM. By using the low voltage mode in a field emission SEM (FESEM) the imaging is possible without coatings. FIG. 2 shows the composite ceramic $\mathrm{BN} / \mathrm{TiB}_{2}$ in material contrast (Sample and FESEM images: Dr.P.Obenaus, Fraunhofer IKTS Dresden, Germany). The slope with $100 \mu \mathrm{m}$ width and several millimetres in length is $10^{4}$ times larger compared with the focused ion beam (FIB) technology. High resolution imaging shows details of the cut in FIG. 3 with the bright $\mathrm{TiB}_{2}$ component, the dark BN and glassy phase. To avoid ion beam induced furrows the sample was rocked within the ion beam cutting plane between $-20^{\circ}$ and $+20^{\circ}$. The cut reveals pores and the interfaces between both components as well as the glassy phase (from the technology) with high quality. Additional ion beam processing is shown in the second example with cutting of Low Temperature Co-fired Ceramics (LTCC) with embedded thermistor structures based on (Mn,Co,Ni,Ru)oxide (Sample: Dr.A.Dziedzic, TU Wroclaw/Poland). After cutting a short-time selective ion etching step was applied to reveal the internal microstructure. FIG. 4 shows the LTCC structure with the distribution of grains in glassy phase after selective etching and AgPd sputter coating taken by a conventional SEM (LaB 6 cathode). FIG. 5 shows bar-like grains transferred from the substrate into the thermistor layer. The modified Gatan Precision Etching Coating System (PECS) allows all processing steps according to FIG.1. The general operating conditions have been described earlier [2]. Here $7 \mathrm{keV}$ $\mathrm{Kr}$ ions with densities up to $20 \mu \mathrm{A} / \mathrm{mm}^{2}$ were applied. A new stage allows sample positioning, tilting and rotation, shield mounting and adjustment with precise control of the sample by optical microscopy or SEM for accurate processing in the PECS. The endpoint determination for processing steps can be controlled by optical in-situ microscopy with the running ion gun or by calibration of the removed material thickness based on measurements and the sputtering behaviour of the investigated materials.

\section{References}

[1] W. Hauffe, Production of Microstructures by Ion Beam Sputtering, Chapter 6 in Sputtering by Particle Bombardment III (Eds. R.Behrisch and K.Wittmaack), Springer Ser. Topics in Applied Physics, Vol. 64 (Springer-Verlag Heidelberg New York (1991) 305

[2] W. Hauffe, D. Gloess, and R.J. Mitro, Microsc. Microanalysis 8, Suppl.2 (2002) 552CD 

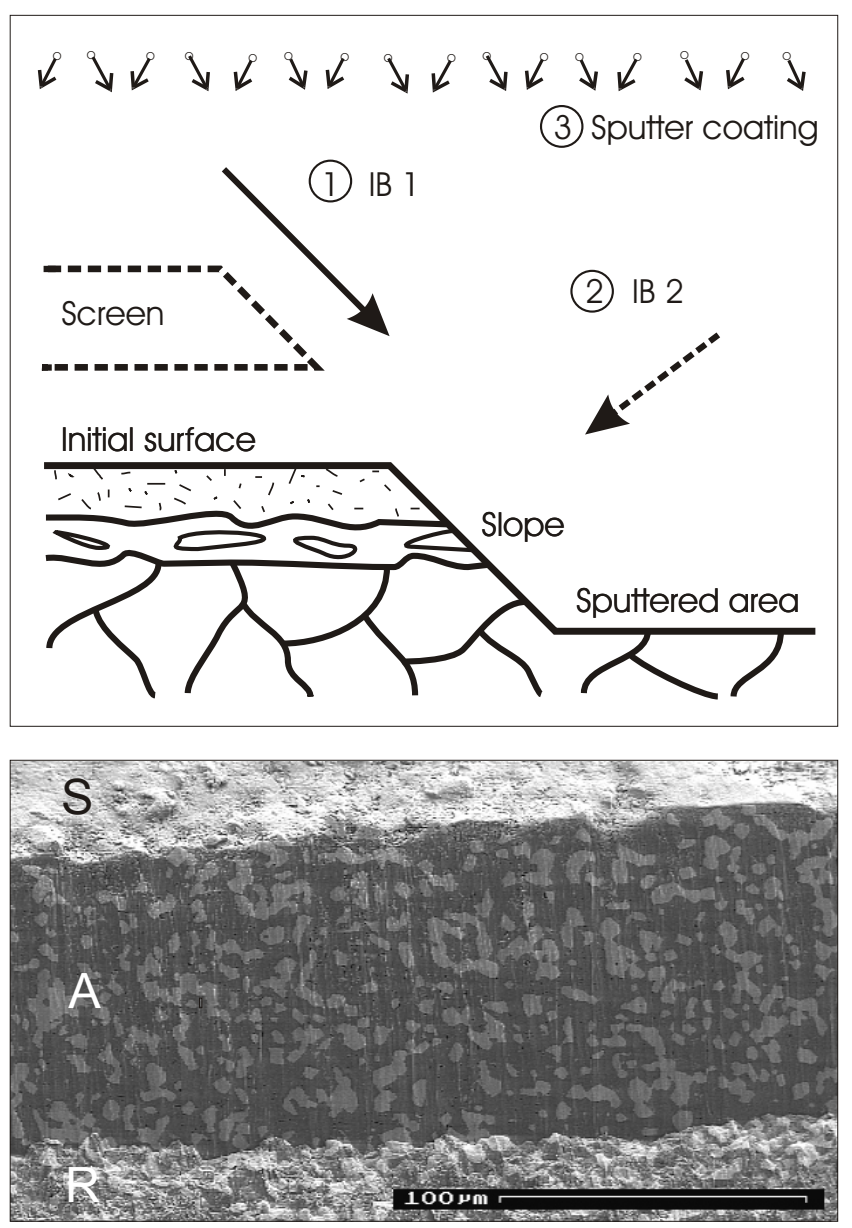

FIG. 2. FESEM image of an ion beam cut through $\mathrm{BN} / \mathrm{TiB}_{2}$ ceramic composite.

S Initial surface; A Slope cut area; R Sputtered region

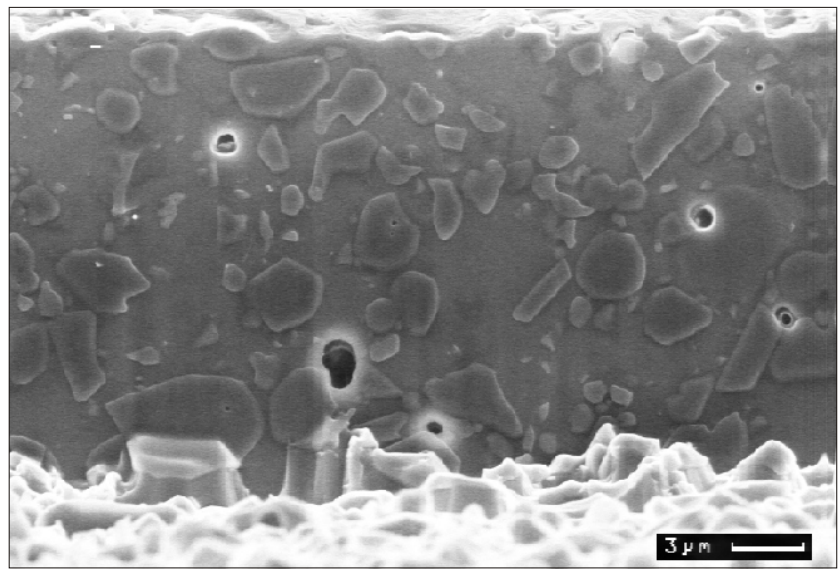

FIG. 4. SEM image of an ion beam cut through the LTCC structure. Distribution of grains and glassy phase (Selective ion beam etching and AuPd coating)
FIG. 1. Ion beam slope cutting (IBSC) for 3D investigation of microstructures with additional ion beam processing steps (selective etching and sputter coating) carried out in Gatan PECS for SEM observation of the initial surface, slope area, and sputtered area

1 IB 1: Ion beam direction for cutting

2 IB 2: Ion beam direction for selective etching and main observation direction of the slope by SEM

3 Sputter coating by ion beam sputtering from selected targets

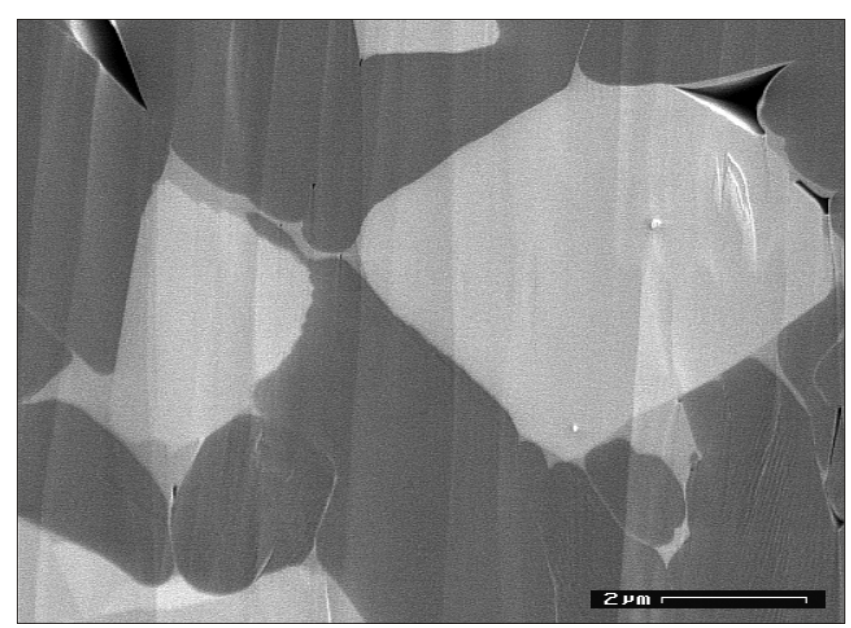

FIG. 3. FESEM image of the ion beam cut area in in FIG. 2 (Detail) with atomic number contrast between the components $\mathrm{BN}$ (dark), $\mathrm{TiB}_{2}$ (bright) and the glassy phase (grey)

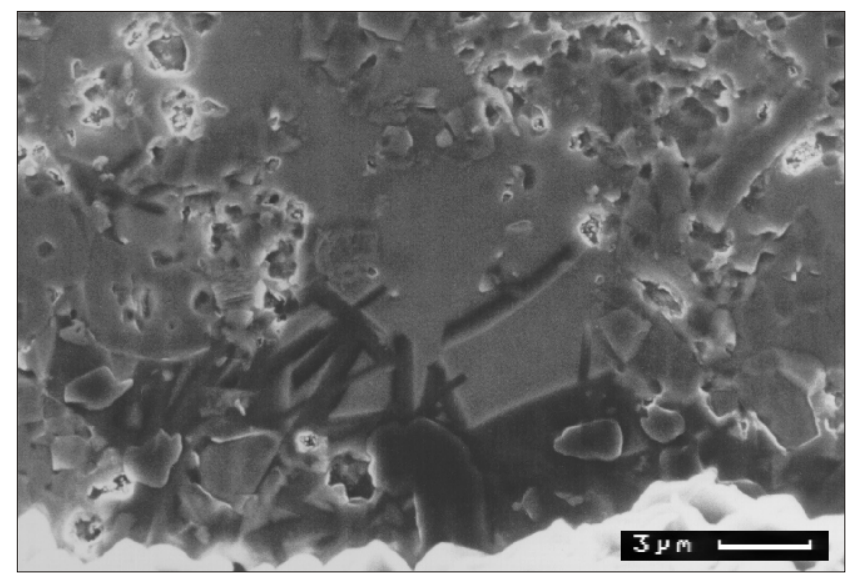

FIG. 5. SEM image of the ion beam cut through the thermistor layer and the substate with bar-like grains (Selective ion beam etching and AuPd coating) 